\title{
Evaluación del desarrollo y crecimiento de Enterolobium cyclocarpum, Maclura tinctoria en condiciones controladas, Estelí -Nicaragua
}

\author{
Mariela Lisbeth Hernández Olivas ${ }^{1}$ \\ Beneyda Leticia Montalván Calderón² \\ Ana María Flores Briones ${ }^{3}$ \\ Josué Tomas Urrutia Rodríguez ${ }^{4}$ \\ Kenny López Benavides ${ }^{5}$
}

\section{RESUMEN}

A pesar de la creciente importancia que tienen los árboles del trópico seco, para la conservación de la biodiversidad, se conoce poco sobre la dinámica de su comportamiento, tales como: su adaptación frente a diferentes cambios del clima. Con el objetivo de aportar al conocimiento de las dinámicas de su comportamiento frente a estos cambios y obtener información sobre las condiciones en las que pueden desarrollarse estas especies. Se evaluó en la Estación Experimental para el estudio del trópico seco "El Limón", el comportamiento en crecimiento y desarrollo de plántulas de las especies Maclura tinctoria y Enterolobium cyclocarpum; sometidas a diferentes dosis de agua y grados de temperatura, contribuyendo así a su recuperación y conservación.Se utilizó un diseño de bloques completamente al azar, haciendo una adaptación de la investigación realizada por Díaz. E (2010).

Los datos obtenidos se analizaron utilizando la prueba de varianza no paramétrica de KruskalWallis. Ninguno de los tratamientos de humedad aplicados en plántulas de Maclura tinctoria mostraron influencia significativa en diámetro al cuello de la raíz y altura, de igual forma ocurrió en altura de E. cyclocarpum, sin embargo en diámetro al cuello de la raíz mostraron diferencias significativas en el tratamiento de Cc más 50\%. Las plántulas de ambas especies expuestas a temperatura ambiente $\left(21.7^{\circ} \mathrm{C}\right)$, mostraron diferencia significativa en diámetro al cuello de la raíz; y en altura presentaron diferencia las de $M$. tinctoria expuestas a $25.7^{\circ} \mathrm{C}$, y en E. cyclocarpum fueron las expuestas a $30^{\circ} \mathrm{C}$. Ambas especies se adaptan a cambios de temperatura y humedad, siendo recomendada en programas de reforestación.

Palabras claves: Crecimiento, desarrollo, Maclura tinctoria, Enterolobium cyclocarpum.

\footnotetext{
1 UNAN-Managua/FAREM-Estelí Correo Electrónico: marielalisbethhernandez@yahoo.es

2 UNAN-Managua/FAREM-Estelí Correo Electrónico: mbeneydaleticia@yahoo.com

3 UNAN-Managua/FAREM-Estelí Correo Electrónico: afloresbriones@yahoo.com

4 UNAN-Managua/FAREM-Estelí Correo Electrónico: josuerod20@yahoo.com

5 UNAN-Managua/FAREM-Estelí Correo Electrónico: kenny.lb@hotmail.com
} 


\title{
Evaluation of the development and growth of Enterolobium cyclocarpum, Maclura tinctoria under controlled conditions, Estelí -Nicaragua
}

\author{
Mariela Lisbeth Hernández Olivas ${ }^{1}$ \\ Beneyda Leticia Montalván Calderón² \\ Ana María Flores Briones ${ }^{3}$ \\ Josué Tomas Urrutia Rodríguez ${ }^{4}$ \\ Kenny López Benavides 5
}

\begin{abstract}
Despite the growing importance of dry tropical trees for the conservation of biodiversity, little is known about the dynamics of its behavior, such as its adaptation to different climate changes. In order to contribute to the knowledge of the dynamics of its behavior to these changes and obtain information about the conditions under which these species can develop, it was evaluated at the Experimental Station for the study of the dry tropics "Limon" the behavior in growth and development of seedlings of the species Maclura tinctoria and Enterolobium cyclocarpum; subjected to different doses of water and degree heat, contributing therefore to its recovery and conservation. It was utilized a randomized complete block design, with an adaptation to the investigation conducted by Diaz. E (2010).

The data obtained were analyzed using the non-parametric test of Kruskal-Wallis variance. None of the moisture treatments applied to Maclura tinctoria seedlings showed significant influence on diameter at root collar and height, just as happened in height to E. cyclocarpum; however, in diameter at root collar it was shown significant differences in the Cc treatment, plus $50 \%$. Seedlings of both species exposed to room temperature $\left(21.7^{\circ} \mathrm{C}\right)$ showed significant difference in diameter at root collar; and in height $\mathrm{M}$. tinctoria exposed to $25.7^{\circ} \mathrm{C}$ presented difference, and E. cyclocarpum that were exposed to $30^{\circ} \mathrm{C}$. Both species are adaptable to changes in temperature and humidity, being recommended in reforestation programs.
\end{abstract}

Keywords: Growth, Development, Maclura tinctoria, Enterolobium cyclocarpum.

1 UNAN-Managua/FAREM-Estelí E-mail: marielalisbethhernandez@yahoo.es

2 UNAN-Managua/FAREM-Estelí E-mail: mbeneydaleticia@yahoo.com

3 UNAN-Managua/FAREM-Estelí E-mail: afloresbriones@yahoo.com

4 UNAN-Managua/FAREM-Estelí E-mail: josuerod20@yahoo.com

5 UNAN-Managua/FAREM-Estelí E-mail: kenny.lb@hotmail.com 


\section{INTRODUCCIÓN}

El crecimiento y desarrollo de las plantas está estrechamente relacionado al medio. La radiación solar es un factor que participa directamente en la fotosíntesis, al igual que el agua, mineral y temperatura. A su vez, todos estos factores afectan el crecimiento, el transporte de nutrientes, la respiración, la transpiración y los procesos que están involucrados en las producciones vegetales.

El estrés hídrico es una condición desarrollada cuando por perdidas excesivas de agua o inadecuada absorción, se produce un balance hídrico negativo suficiente para reducir la turgencia, disminuir la elongación celular y alterar negativamente los procesos fisiológicos esenciales de las plantas. Además varían en función de la especie y con su grado de tolerancia, pero también en función de la magnitud de la falta de agua y de la rapidez con que se experimente la carencia de la misma. El grado de endurecimiento de una planta de vivero es un factor esencial a la hora de definir su aptitud para ser trasplantada al campo. Cuanto mayor sea ese grado de endurecimiento, mayor será la capacidad para soportar el estrés producido durante la plantación, el estrés postrasplante y las heladas. El proceso de endurecimiento viene controlado por factores ambientales como la temperatura y el fotoperiodo (Villa - salvador et al. 1999).

El presente estudio tiene como propósito evaluar el desarrollo y crecimiento de plántulas bajo condiciones controladas de temperatura y humedad. Esto permitirá conocer las condiciones en que se puede desarrollarlas plántulas, y si existen variaciones en diámetro y altura al ser expuestas a estas condiciones, todo esto a fin de contribuir a su conservación,

El estudio dará pautas para el desarrollo de programas de investigación, reproducción y preservación de estas especies, ya que en la actualidad son pocos los estudios que se han realizado a pesar de la importancia que representan estas especies en los ecosistemas.

\section{MATERIALES Y MÉTODOS}

\section{Ubicación del área de estudio}

La Estación Experimental se encuentra ubicada dentro de la zona de amortiguamiento de Área Protegida El Tisey - La Estanzuela, La Estación se encuentra a $1.5 \mathrm{~km}$ al suroeste de la ciudad de Estelí. Este sector pertenece según Holdrich, al Bosque seco Tropical, con temperaturas promedio de $24-26^{\circ} \mathrm{C}$, comprendidas entre los meses de mayo y octubre, con un periodo canicular de julio a agosto, una precipitación media anual que oscila entre $800-900 \mathrm{~mm}$, su ubicación geográfica se encuentra entre las coordenadas UTM 0568720 y 1443707 y una altura de 800 y 884 msnm (Zeledón, 2004).

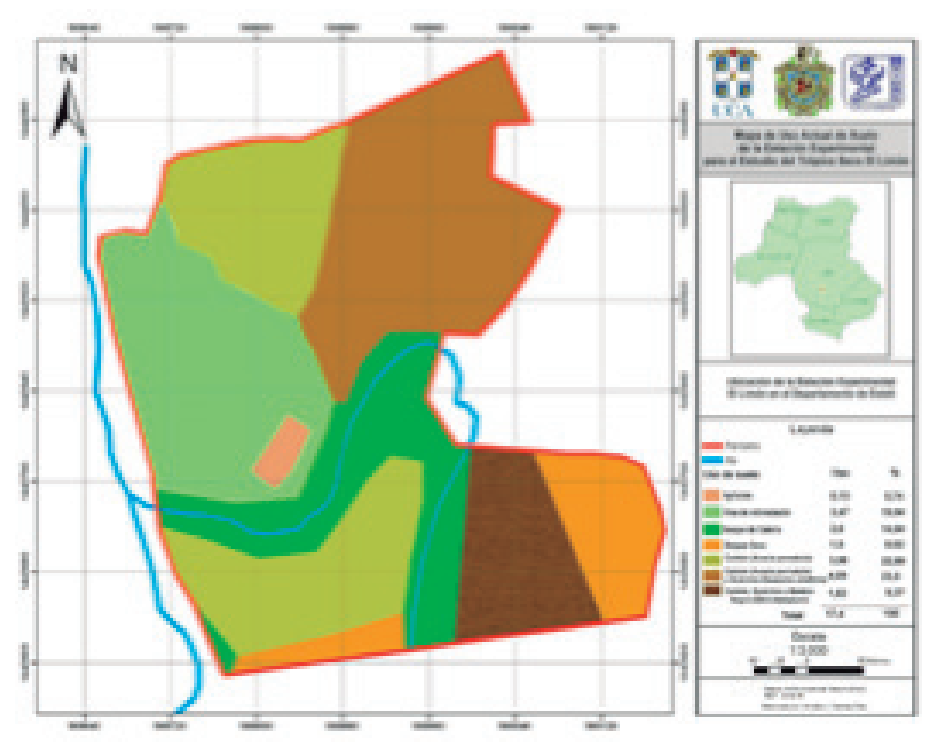

Figura 1: Estación Experimental "El Limón”. 
Para la evaluación del comportamiento en crecimiento y desarrollo de plántulas en condiciones controladas de temperatura y humedad, se recolectaron semillas de las dos especies. Posteriormente se realizó una prueba de germinación con el objetivo de comprobar la viabilidad de la semilla y de esta manera garantizar el porcentaje de germinación. Para la estimulación de la germinación se aplicaron tratamientos pregerminativos diferenciados para cada especie a fin de acelerar el proceso de germinación; tomando en cuenta las características de las semillas, que van desde la escarificación mecánica de la testa (E. cyclocarpum), hasta hidratación en agua fresca durante 24 horas (M. tinctoria).

Posteriormente se procedió al establecimiento del experimento en el invernadero. Se llenaron un total de 630 bolsas de polietileno de tres libras cada una, utilizando para el llenado sustrato compuesto de: $75 \%$ de tierra y $25 \%$ de arena, desinfectado con agua hirviendo ( $100{ }^{\circ} \mathrm{C}$ Punto de Ebullición) para evitar problemas fitosanitarios. Las semillas se sembraron directamente, colocando tres por cada una de las bolsas. Se utilizó un diseño de Bloques Completamente al Azar (BCA) (Piura, L. J. 1998). El cual permite distribuir los controles y los tratamientos experimentales estratificadamente. El diseño utilizado para los experimentos de temperatura y humedad es una adaptación de la investigación realizada por Díaz E. (2010), adaptado de acuerdo a los tratamientos.

\section{Tratamiento de temperatura aplicados en plántulas de Maclura tinctoria y Enterolobium cyclocarpum.}

Para la realización de este experimento se contó con el registro de datos meteorológicos de la Estación experimental "El Limón", indicando que para los meses de noviembre 2012 a febrero 2013 la temperatura promedio fue de $21.7^{\circ} \mathrm{C}$, temperatura utilizada como referencia para los tratamientos dentro del invernadero. Se realizó un experimento previo en invernadero tomando datos de temperaturas cada tres días,en tres lapsos de tiempo, durante un mes; obteniendo un promedio de temperatura dentro del invernadero de $25^{\circ} \mathrm{C}$. Posteriormente se realizó otro preexperimento para comprobar si el número de capas de plástico a colocar aumentaría o disminuiría la temperatura con respecto a la registrada en invernadero; realizándose durante cinco días consecutivos. A partir de los resultados obtenidos se definieron los siguientes tratamientostemperatura:

Testigo o control: Fuera del invernadero condiciones naturales $\left(21.7^{\circ} \mathrm{C}\right)$.

Tratamiento 1: Invernadero $\left(25^{\circ} \mathrm{C}\right)$.

Tratamiento 2: Invernadero más una capa de sombra $\left(27.5^{\circ} \mathrm{C}\right)$.

Tratamiento 3: Invernadero más dos capas de sombra $\left(30^{\circ} \mathrm{C}\right)$.

Se utilizó una población de 360 plántulas para las dos especies (180 plántulas por cada especie).

Se establecieron tres repeticiones (conformados por 15 plántulas) por cada uno de los tratamientos de temperatura, con un total de 45 plántulas por cada tratamiento; además se utilizó un testigo referente fuera del invernadero con 45 plántulas por especie. Las plántulas de este tratamiento estuvieron sometidas a riego a dosis de capacidad de campo.

\section{Tratamientos de humedad aplicadosen plántulas de Maclura tinctoria y Enterolobium cyclocarpum}

Se procedió a la determinación de la capacidad de campo de 8 bolsas (Cada bolsa contiene 300 gr de muestra) saturando el sustrato y dejando escurrir el excedente de agua por 24 horas, transcurrido este tiempo se pesaron para obtener el peso fresco, posteriormente se llevaron al horno a una temperatura de $100^{\circ} \mathrm{C}$ durante 24 horas, obteniendo el peso seco. A partir de la diferencia del peso promedio entre ambos se determinaron las dosificaciones de agua para las plántulas de acuerdo al tratamiento, estableciendo el riego cada 4 días (Porta et al 1999). 
T1 Ó Testigo: Capacidad de campo $=576 \mathrm{ml}$

T2: Capacidad de campo menos $50 \%=288 \mathrm{ml}$

T3:Capacidad de campo más $50 \%=864 \mathrm{ml}$

Para este tratamiento de humedad se utilizó una población de 270 plántulas para las dos especies (135 plántulas por cada especie).

Por cada tratamiento se establecieron tres repeticiones conformadas por 15 plántulas cada una, para un total de 45 plántulas por cada tratamiento.La aplicación de los tratamientos se llevó a cabo cada cuatro días (Boby Moncada. et. al. 2005).

Para el registro de las mediciones realizadas se le asignó a cada plántula un código según el tratamiento utilizado. Se realizaron medidas de altura con el uso de regla $(\mathrm{cm})$, y diámetro al cuello de la raíz con el uso del pie de rey ó Vernier ( $\mathrm{cm} / \mathrm{mm})$, las mediciones se iniciaron a partir de la emergencia de hojas verdaderas de las plántulas. La toma de datos se llevó a cabo cada 15 días durante un periodo de tres meses (Diciembre 2012 a Febrero 2013).

\section{Análisis estadístico}

Se determinó la normalidad de los datos, utilizando la prueba estadística de Kolmogorov - smirnov (KS) para muestras independientes. Posteriormente se realizaron pruebas de normalización de datos, a través de logaritmos naturales, raíz cuadrada y arco seno de la raíz cuadrada. Después se aplicaron Análisis de Varianza no Paramétricos de Kruskall Wallis.

Para el análisis de datos se utilizaron los software: Excel versión 2013, SPSS versión 20 e InfoStat versión 2013.

\section{RESULTADOS Y DISCUSIÓN}

Desarrollo y crecimiento de plántulas bajo condiciones controladas de humedad y temperatura en invernadero.

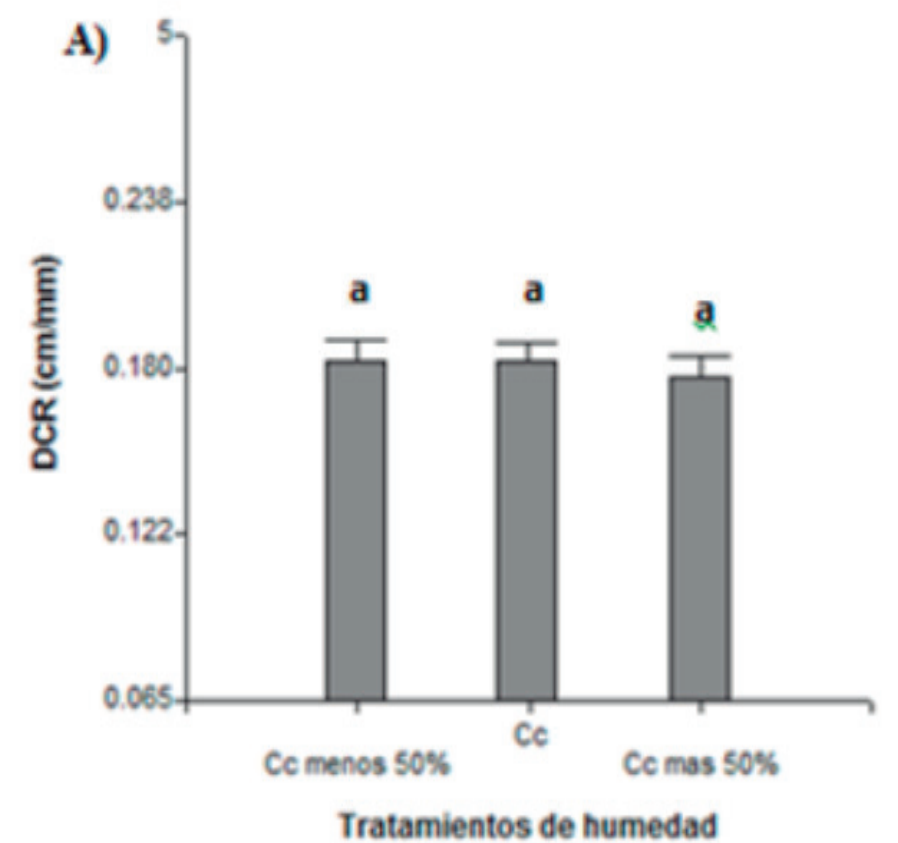

Figura A: Diferencias del diámetro al cuello de la raíz al aplicar tratamientos de humedad en plántulas de Maclura ticonora

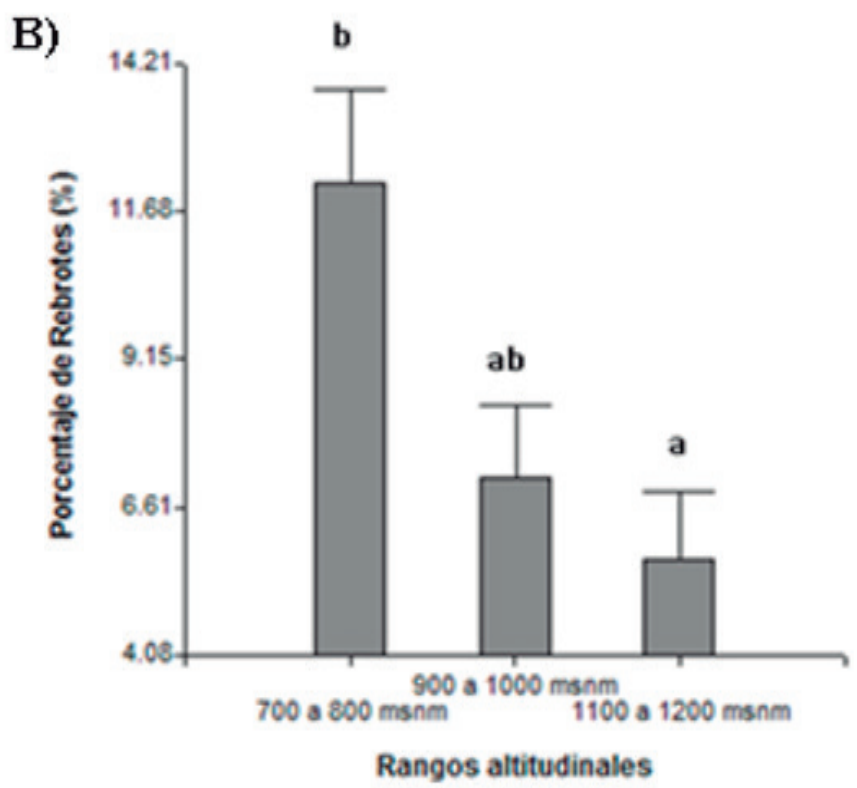

Figura A: Diferencias del diámetro al cuello de la raíz al aplicar tratamientos de humedad en plántulas de Maclura ticonora

Figura 1. Características fenológicas de la especie Maclura tinctoria: porcentaje de hojas (A) y rebrotes (B), a tres rangos altitudinales. 
Maclura tinctoria es una especie semi-decidua es decir que deja caer un alto porcentaje de sus hojas durante la temporada seca lo cual coincide con la producción de flores, convirtiéndose así en un indicador del inicio de esta fenofase. Según Scott (1966) una gran mayoría de los árboles que habitan estas regiones con períodos de sequía prolongados están, en una u otra forma, adaptados a estas condiciones ambientales.

Es preciso destacar que la aparición de rebrotes y presencia de hojas durante los meses estudiados (abril a octubre) permaneció de manera irregular entre los individuos por rango altitudinal; ya que el brote de hojas inicia de manera casi simultánea con la caída, por lo que no se alcanzan a observar durante mucho tiempo al individuo sin follaje y la mayor producción de hojas nuevas se registra durante la temporada de lluvias, esto es, una vez que finaliza la cosecha de los frutos y continúa durante buena parte del año.

Sin embargo autores como Borchet (1994), asignan el papel más importante en el control de la duración de la fase de aparición de rebrotes y presencia de hojas a la interacción entre la edad de estas hojas y el inicio de la estación seca.

Scott (1966) reporta que la fenología de los árboles tropicales puede responder también a señales ambientales. Sin embargo autores como Reich y Borchert (1984), señalan que algunos de estos comportamientos son facultativos debido a que individuos de la misma especie que crecen en sitios diferentes o en el mismo sitio pueden desplegar marcadas diferencias fenológicas entre sí.

\section{Porcentaje de flores y frutos}

No se encontraron diferencias significativas $(\mathrm{P}>0.3260$, $\mathrm{ns}$ ), en el porcentaje de flores; los individuos de la especie Maclura tinctoria encontrados en los tres rangos altitudinales de 700 a $800 \mathrm{msnm}, 900$ a $1000 \mathrm{msnm}$ y 1100 a $1200 \mathrm{msnm}$, no presentaron variaciones en la característica fenológica (Ver figura, C).
Sin embargo en la característica de fructificación si mostraron diferencia significativa $(\mathrm{P}<0.0497)$; indicando que los individuos del rango altitudinal de 700 a 800 msnm presentan un mayor porcentaje de frutos, con respecto a los demás rangos (Ver figura, D).
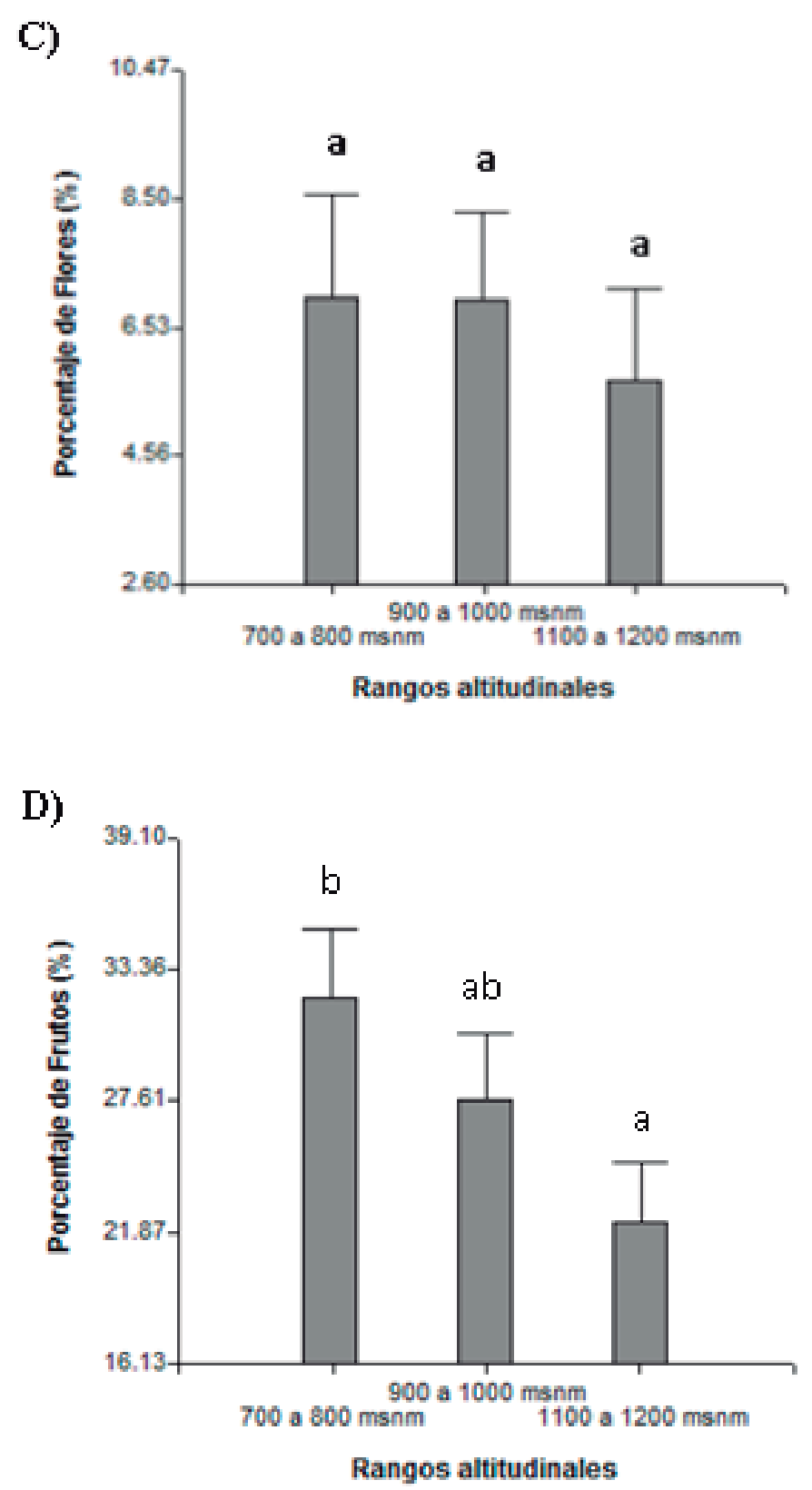

Figura 2. Características fenológicas de la especie Maclura tinctoria: porcentaje de flores (C) y frutos (D) en tres rangos altitudinales. 
Los resultados obtenidos reflejan que los individuos de Maclura tinctoria a diferentes rangos altitudinales muestran comportamiento fenológico diferentes; observándose que la parte baja es la que presenta mayores porcentajes en las etapas de fructificación, hojas y rebrotes con respecto a la zona media y alta.

A Scott (1966) le parece un tanto paradójico que, la mayor floración se encuentre concentrada en la época seca, que lógicamente debe ser la más crítica para las plantas. Esta observación de Scott es muy atinada; sin embargo, hay algunos hechos que parecen sugerir que la concentración de floración en esta época es más bien el resultado de un largo proceso de adaptación, parte de la misma dinámica de estas formaciones. Estos resultados están relacionados al éxito reproductivo y es posible que esta especie pueda producir más frutos en las zonas bajas que en las altas, porque los individuos que se encuentran ahí responden mejor a las condiciones bióticas y abióticas del ambiente.

Según lo que expresa Gómez (2010), el análisis de los períodos de ocurrencia de las etapas fenológicas de las especies en las zonas bajas exhibe una mayor estacionalidad que las especies de las zonas altas esto corrobora lo observado en el estudio; esto no quiere decir que se esté produciendo una mejor adaptación a menores alturas, ya que la especie es propia de un hábitat de alturas a nivel del mar y los 1,200 msnm (Zamora, N.1999).

Algunos estudios realizados de fenología de las especies, encontraron que de manera general, la identificación de los patrones de fructificación se torna difícil en algunas especies, ya sea porque los frutos de la misma cosecha permanecen en el árbol por largo tiempo; o por el contrario, desaparecen rápidamente de la copa del árbol porque son muy apetecibles para la fauna silvestre, o porque son abortados, por lo que existe variación en la producción de frutos en los diferentes individuos de la misma especie, y que ésta depende de la intensidad y la duración de la estación seca y de las formas de vida (Foster, 1990).
De igual forma se considera lo expresado por Mejía (1990), quien indicó que la altitud, influye en la fenología de las plantas, nuestros resultados confirman lo expresado por Mejía, porque se corroboró que los individuos de la especie Maclura tinctoria al encontrarse en diferentes altitudes presentan variación en sus etapas fenológicas. Por su lado Borchert (1996), analizó las diferencias fenológicas de 18 especies tropicales mediante el uso de colecciones de herbario, y encontró que el ámbito de la variación geográfica tiene un rol importante en el comportamiento de las especies y destacó que las diferencias en la duración y la intensidad de la época seca afectan mucho la sincronía de la floración, y por ende la de la fructificación.

Comportamiento fenológico de la especie arbórea Enterolobium cyclocarpum Jacq en tres rangos altitudinales

\section{Porcentaje de hojas y rebrotes}

Existe diferencia significativa en el porcentaje de hojas en los individuos de Enterolobium cyclocarpum, ya que el valor de significación es de $\mathrm{P}<0.0011$; el rango de 900 a $1000 \mathrm{msnm}$ presenta el mayor porcentaje de la característica respecto al rango de 700 a $800 \mathrm{msnm}$ que presentó los valores más bajos; en cambio el rango de 1100 a $1200 \mathrm{msnm}$ presenta características similares de los demás rangos (Ver figura, E). En la etapa de rebrotes también se presentó diferencia significativa $(\mathrm{P}<0.0002)$; los rangos de 900 a $1000 \mathrm{msnm}$ y 1100 a $1200 \mathrm{msnm}$ presentan los mejores porcentajes de rebrotes, respecto al de 700 a 800 msnm que alcanzó los valores más bajos (Ver figura, F). 

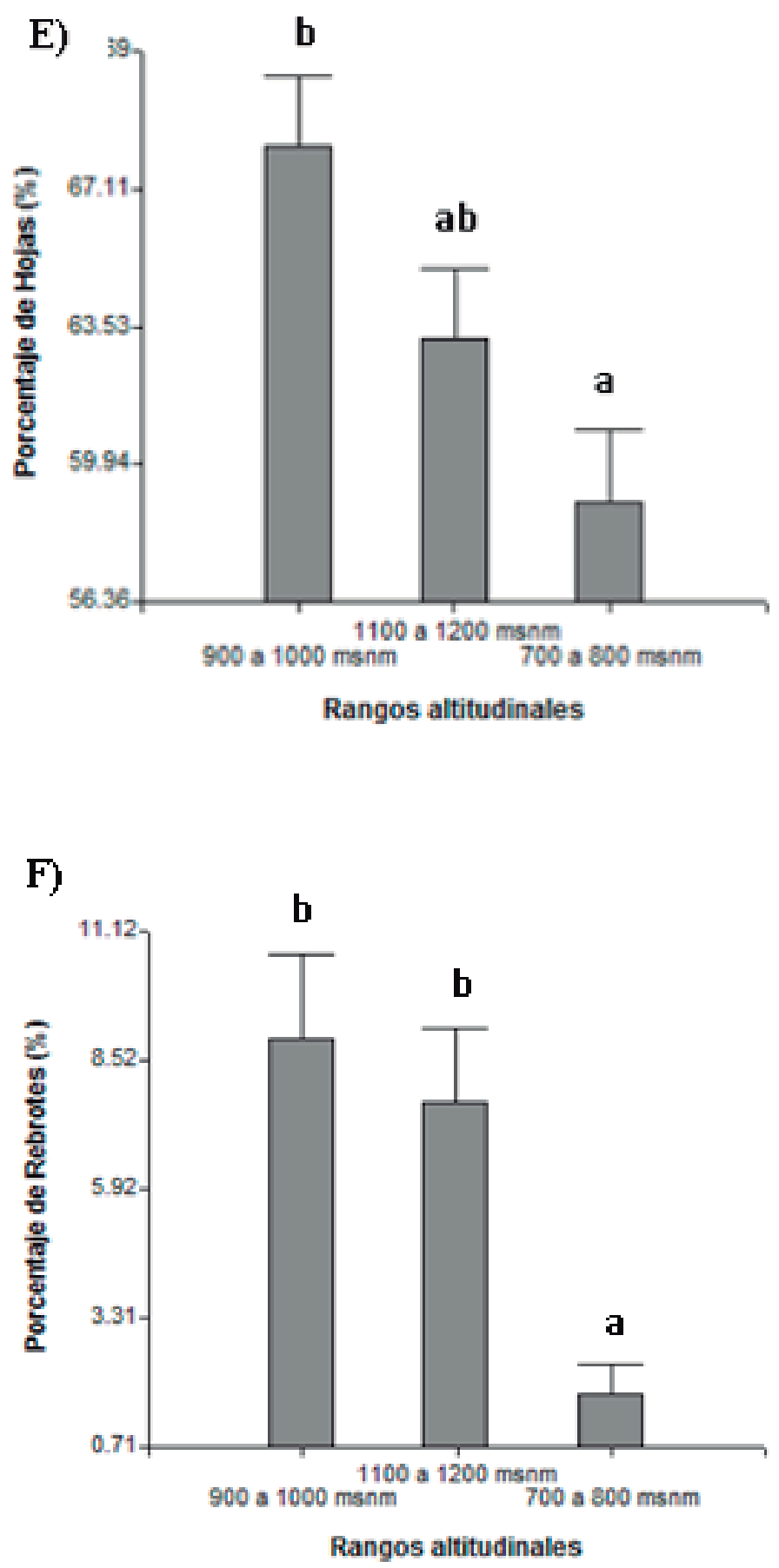

Figura 3. Características fenológicas de la especie Enterolobium cyclocarpum: porcentaje de hojas (E) y rebrotes $(\mathrm{F})$ en tres rangos altitudinales.
El Guanacaste coloniza una gran variedad de hábitat, a pesar de que es común a elevaciones bajas se le puede encontrar creciendo de manera natural hasta los 900 msnm y se le ha plantado a elevaciones de $1100 \mathrm{msnm}$. Es un árbol semi-caducifolio, es decir que no pierde las hojas en su totalidad. Su defoliación está altamente asociada con la floración y la formación de los frutos, eventos que a su vez se presentan en los meses más secos del año.

Es una especie que presenta patrón fenológico de copa, por lo que tiene la sorprendente capacidad de producir y expandir el follaje antes de la caída de las primeras lluvias, denominándose especie de brotación temprana (Frankie et al. 1974), comportamiento ya expresado por Richards (1996), quienes comentan que una gran proporción de árboles de bosques tropicales nunca están desnudos de hojas y que la caída de hojas viejas es rápidamente seguida por la aparición de hojas jóvenes. Caso que sucede en la especie Enterolobium cyclocarpum que la caída de hojas viejas es acompañada por hojas nuevas o rebrotes.

La brotación de las hojas en esta especie es acompañada por la apertura de las flores y la formación temprana de frutos, los cuales luego interrumpen su desarrollo durante la estación lluviosa (Rojas et al. 2007). Existen algunas hipótesis que señalan los cambios en el contenido de humedad del suelo (Reich \& Borchert 1984), el fotoperiodo (Bullock 1990), la temperatura (Gómez \& Fournier 1996) y ritmos endógenos (Fournier \& Fournier 1986), como posibles activadores de la brotación temprana y a las reservas internas de agua como las fuentes que soportan la brotación del follaje (Borchert 1994b). 
Autores como Orozco (1992) señalan que muchas especies pueden tener comportamientos distintos en diferentes sitios, caso que se presentó en las etapas de hojas y rebrotes presentando diferencia significativa principalmente en el rango altitudinal de 900 a 1000 msnm, respecto a los demás rangos.

Porcentaje de flores y frutos

No existen diferencias significativas en el porcentaje de flores y frutos, los individuos de la especie Enterolobium cyclocarpum, ya que el valor de significación es $(\mathrm{P}>0.05)$, no se presentó variación alguna en la característica en ninguno de los rangos (Ver figura, G, H).

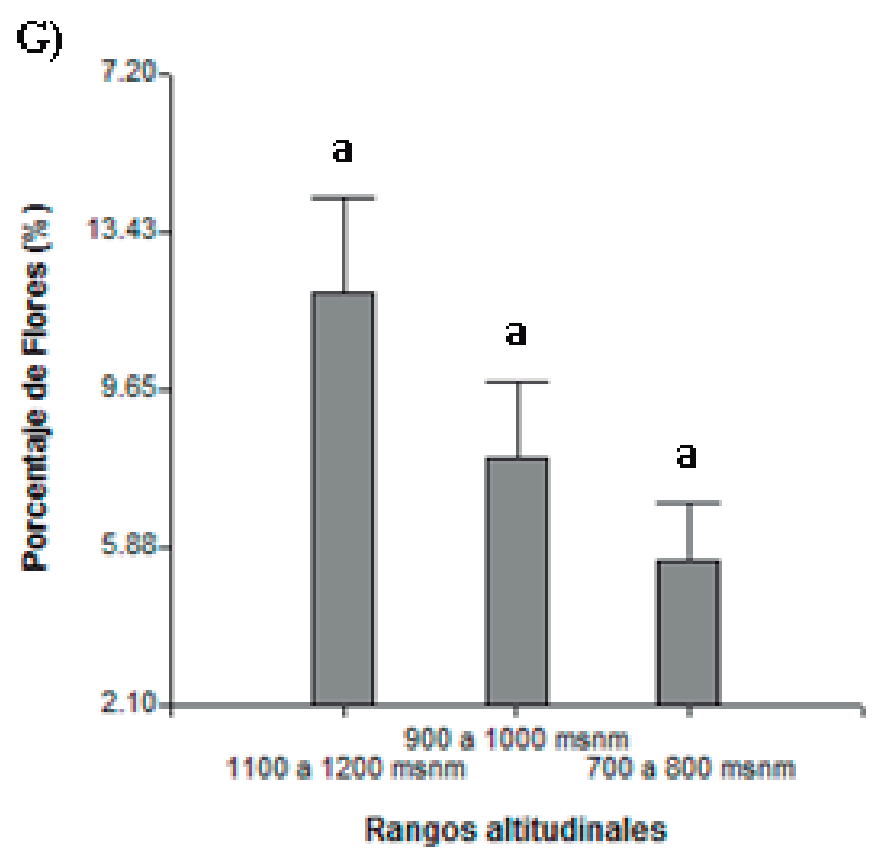

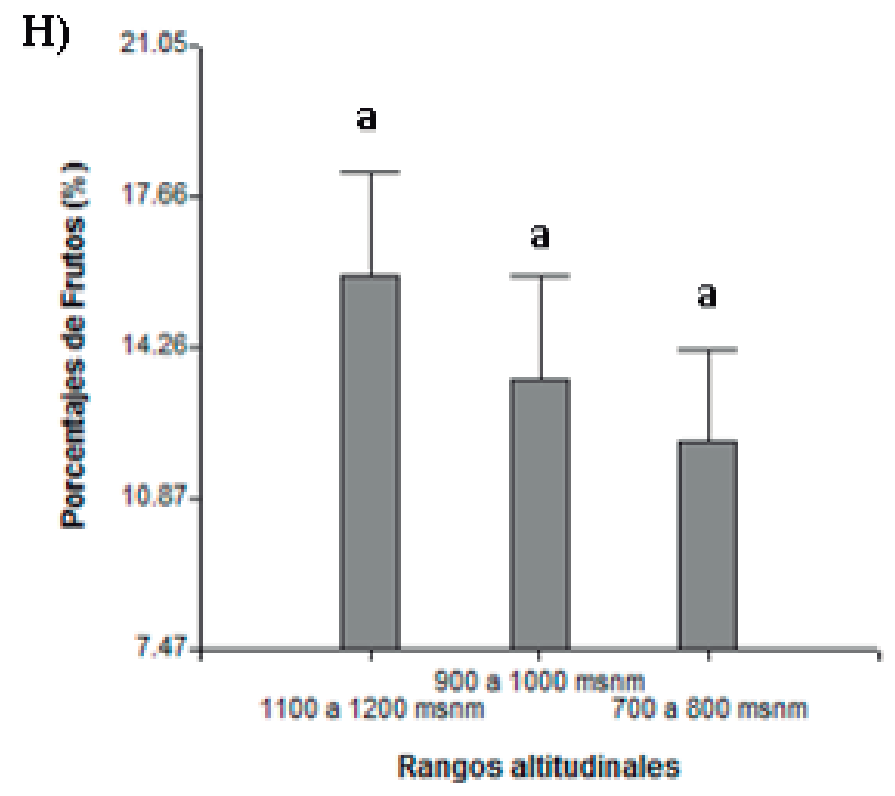

Figura 4: Características fenológicas de la especie arbórea Enterolobium cyclocarpum: porcentaje de flores $(\mathrm{G})$ y frutos $(\mathrm{H})$, en tres rangos altitudinales.

Según Blaser y Camacho (1991), la ausencia de diferencias significativas entre sitios en la mayoría de las especies demuestra que estas tienen comportamientos fenológicos similares en un área determinada. Esto se puede relacionar con lo encontrado en las etapas de floración y fructificación de los individuos de la especie Enterolobium cyclocarpum que en los tres rangos altitudinales no presentaron diferencia significativa alguna.

Asimismo, Aide (1988) considera que las especies al desarrollar la floración y la fructificación pierden algo de su follaje, por lo tanto, la caída del follaje favorece la floración, caso contrario al que sucede con las especie Maclura tinctoria y Enterolobium cyclocarpum que mantiene su follaje al mismo tiempo que florecen y fructifican. Por otro Pires-O'Brien (1995), destaca que la falta de lluvia, podría el principal factor responsable de que los árboles florezcan en períodos secos, evitando la pérdida masiva de flores. 
La floración y fructificación son características relevantes, que determina el rendimiento reproductivo de las especies, ya que puede poner restricciones en el uso de los recursos estacionales, tales como luz, agua y polinizadores por parte de las plantas (Marco et al., 2000). La mayoría de las especies de los bosques tropicales presentan variación estacional en la aparición de nuevas hojas, flores y frutos; este patrón sugiere que los cambios fenológicos representan adaptaciones a factores bióticos y/o abióticos, siendo el clima el factor principal (Van Schaik et al., 1993).

Borchert (1980) descarta los factores climáticos como determinantes primordiales de la floración de las especies arbóreas en los trópicos y considera que los patrones fenológicos son determinados, principalmente, por procesos periódicos endógenos y en forma secundaria como adaptación a cambios ambientales.

Las diferencias encontradas en el comportamiento fenológico y en la productividad de la especie respecto a otros estudios (Vélez, 1992) puede deberse a metodologías diversas, ya que la duración de tales estudios (menos de 3 años) pudo no permitir la clara identificación del ciclo reproductivo. De acuerdo con Newstron et al. (1994), como muchas especies presentan ciclos fenológicos multianuales, se requieren períodos de observación prolongados, por lo menos 5 años, para describir completamente estos patrones.

\section{CONCLUSIONES}

Los individuos de la especie Maclura tinctoria mostraron diferencias significativas en el porcentaje de hojas, rebrotes y frutos; en la zona baja (700 a $800 \mathrm{msnm}$, respecto a las demás zonas; a diferencia de las flores. En cambio en Enterolobium cyclocarpum mostraron diferencia significativa los individuos de la zona media (900 a 1000) en el porcentaje de hojas y rebrotes; las etapas de floración y fructificación, no mostraron diferencia significativa alguna.

\section{BIBLIOGRAFÍA}

Aide, T.M. (1988). Herbivory as a selective agent on the timing of leaf production in a tropical understory community. Nature. Vol. 336; p. 574-575.

Betancourt, A. (1987). Silvicultura especial de árboles maderables tropicales. Cuba. Científico Técnica. p. 342-356.

Borchert, R (1980). Phenology and ecophysiology of tropical trees: erythrina poeppigiana O.F. Cook. Ecology 61: 1065- 1074.

Borchert, R. (1994) b. Soil and stem water storage determine phenology and distribution of tropical dry forest trees. Ecology 75(5):1437-1449.

Borchert R (1996). Phenology and flowering periodicity of Neotropical dry forest species: evidence from herbarium collections. J. Trop. Ecol. Vol. 12; p. 65-80.

Blaser, J. \& M. Camacho. (1991). Estructura, composición y aspectos silviculturales de un bosque de roble (Quercus spp.) del piso montano en Costa Rica. CATIE, Turrialba, Costa Rica. 68p.

Bullock, S; Solís-Magallanes, A. (1990). Phenology of canopy trees of a tropical deciduos forest in México. Biotrópica 22(1):22-35.

Chuine, I., and E. Beaubien. (2001). Phenology is a major determinant of temperate tree range. Ecology Letters 4:500-510. 
Frankie, G; Beker, H; Opler, P.1974. Comparative phenological studies of trees in tropical wet and dry forests in the lowlands of Costa Rica. Journal of Ecology. 62:881-919 Forest Service. 1943. The forests of Costa Rica. Washington, DC: U.S. Department of Agriculture. 48 p.

Foster R. (1990). Long-term change in the successional forest community of the Rio Manu Floodplain. In: A.H. Gentry ed. Four Neotropical rainforests. Yale Univ. Press, New Haven, CT.: 565-572.

Fournier, L. y C. Charpantier. (1975). El tamaño de la muestra y la frecuencia de las observaciones en el estudio de las características fenológicas de los árboles tropicales. Turrialba 25: 45-48.

Fournier, L; Fournier, M. (1986). Fenología y ecofisiología de Gliricidia sepium "Madero Negro" en Ciudad Colón, Costa Rica. Rev. Biol. Trop. 34(2):283-288.

Gómez, P; Fournier,L.(1996). Fenología y ecofisiología de dos poblaciones de Tabebuia rosea ("Roble de Sabana") en Costa Rica (Bignoniaceae). Rev. Biol. Trop. 44(1):61-70.

Gómez R. Martha Ligia (2010). Fenología reproductiva de especies forestales nativas presentes en la jurisdicción de CORANTIOQUIA, un paso hacia su conservación. Volumen I /, Corporación Autónoma Regional del Centro de Antioquia, CORANTIOQUIA. Medellín: CORANTIOQUIA, 228 p.ISBN: 978-958-99363-3-7.

Marco, D.E., A.A. Calviño and Sergio Páez. (2000). Patterns of Flowering and Fruiting in Papulations of Larrea divaricata in Dry Chaco (Argentina). Journal of Arid Environments 44:327-346.

Mejía, M.G. (1990). Fenología: Fundamentos y métodos. En. Seminario Taller en Semillas Forestales Tropicales. (2 ${ }^{\circ}$ Bogotá, Colombia). Memoria. Ed. T. Triviño. Bogotá, CO. p. 65-79.

Newstrom, L.E., Frankie, G.W. y Baker, H.G. (1994). A new classification for plant phenology based on flowering patterns in lowland tropical rain forest trees at La Selva, Costa Rica. Biotropica, 26(2): 141-159.
Orozco, L. (1992). Estudio ecológico y de estructura horizontal de seis comunidades boscosas de la Cordillera de Talamanca, Costa Rica. CATIE, Turrialba, Costa Rica. 33 p.

Pires-O'Brien, M.J.; O’Brien, C.M. (1995). Aspectos evolutivos da fenología reprodutivadas árvores tropicais. Belém: FCAP, 25 p.

Preuhsler, T., A. Bastrup-Birk \& E. Beuker. (2006). Manual on methods and criteria for harmonized sampling, assessment, monitoring and analysis of the effects of air pollution on forests Part IX Phenological Observations. United Nations Economic Commission for Europe Convention on Long-Range Transboundary Air Pollution. International Cooperative Programme on Assessment and Monitoring of Air Pollution Effects on Forests. Consultada el 22 de agosto del 2006, (http://www.metla.fi/eu/icp/phenology/ manual).

Reich, P.B, Borchert, R (1984). Water stress and tree phenology in a tropical dry forest in the lowlands of Costa Rica. Journal of Ecology 72: 61-74.

Richards, P. W. (1996). The tropical rain forest. Cambridge University Press. Cambridge, U.K. 575 pp.

Rojas-Jiménez, K; Hoolbrok, NM; Gutiérrez, M. (2007). Dry-season leaf flushing of Enterolobium cyclocarpum (ear-pod tree): aboveand belowground phenology and water relations. Tree Physiology 27:1561-1568.

Scott, N. (1966). Ecologically importa11l aspects 01 the climates of Costa Rica, Organization for Tropical Studies, Costa Rica, 26 pp. (mimeografiado).

Van Schaik, C; Terborgh, J. W. y Wright, S. J. (1993). The phenology of tropical forests: adaptative significance and consequence for primary consumers.Annu. Rev. Ecol. Syst. 24: 353-377.

Vélez, G. (1992). Estudio fenológico de diecinueve frutales silvestres utilizados por las comunidades indígenas de la región de Araracuara - Amazonía colombiana. Colombia Amazónica, 6: 135-186. 
Zamora, N.; González J. \& Poveda, L. J. en prep. (1999). Árboles y Arbustos del Bosque Seco de Costa Rica. Instituto Nacional de Biodiversidad, Costa Rica.
Zeledón Alba Marina (2002). Estructura del bosque tropical seco y sus usos, en las comunidades de El Limón, el Dorado y el Coyolito - Estelí, Estelí marzo 2004. Universidad Autónoma de Barcelona (UAB - FAREM). 